\title{
Probing disk inhomogeneities using spectropolarimetry in the extreme binary epsilon Aurigae
}

\author{
Kathleen Geise and Robert Stencel \\ University of Denver, \\ 2112 East Wesley Avenue, Denver, Colorado 80208, USA \\ email: kgeise@du.edu
}

\begin{abstract}
The $\varepsilon$ Aurigae system is a single-line spectroscopic binary system that consists of a variable F0 supergiant star and an occulting disk surrounding an unseen object, probably a B star. The eclipse occurs every 27 years and lasts for almost 2 years. Spectral features attributed to the disk exhibit line shifts due to the rotation of the disk that are easily observed in spectra. We obtained 50+ epochs of high dispersion optical spectropolarimetric data from the ESPaDOnS instrument at the Canada-France-Hawaii Telescope before, during, and after the most recent eclipse (2009-2011). We found numerous 3-sigma (or greater) linear polarization features in the spectra and associated these with atomic absorption features also present in the spectra. We observed dramatic changes to polarization and position angles with time during eclipse, particularly around $3^{\text {rd }}$ contact. The increased polarization could be due to a localized increased number of scatterers.
\end{abstract}

Keywords. binaries: eclipsing, line: identification, polarization, accretion disks

\section{Introduction}

$\varepsilon$ Aurigae is a single line spectroscopic binary system comprised of a variable F0 supergiant star (the visible star in the system) and a dusty disk surrounding a presumed B star (Fig. 1). The F0 star is sometimes characterized as an irregularly variable star or a non-radial pulsator. The system eclipses once every 27 years for a duration of two years, among the longest periods of totality for known eclipsing systems. Many characteristics of $\varepsilon$ Aurigae are still poorly defined, including the mass of each object, spectral type of the binary companion, origin of the disk, and evolutionary status of the system, largely because the distance is uncertain. An ultraviolet excess in the spectral energy distribution suggests the second object is a B5 star (Hoard et al. 2010). We have made use of spectropolarimetry in order to improve knowledge of the components in this system.

\section{Observations \& Methods}

More than 50 high resolution spectra were obtained from the Canadian Data Center (CADC) archives for the Echelle SpectroPolarimetric Device for the Observation of Stars (ESPaDOnS) instrument at the Canada-France-Hawaii telescope (CFHT). ESPaDOnS is a cross-dispersed echelle spectropolarimeter designed to obtain a complete optical spectrum (370 nm to $1000 \mathrm{~nm}$ ) in a single exposure, with a resolving power of about 65,000 and signal-to-noise greater than 500 (Donati 2003). Observations were taken by Nadine Manset as the principal investigator, before, during and after the most recent eclipse. 


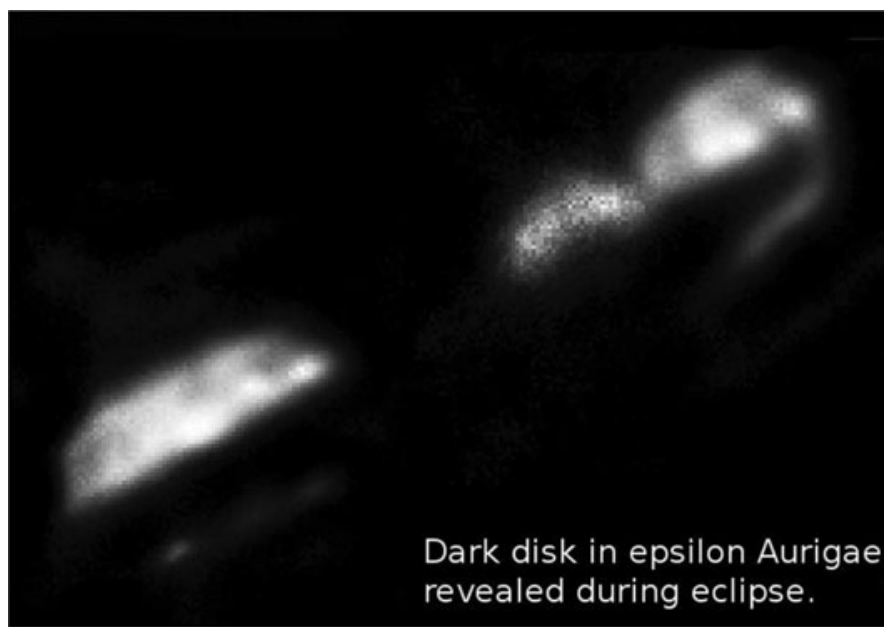

Figure 1. The disk of $\varepsilon$ Aurigae in a composite of interferometric images. Image courtesy of B. Kloppenborg \& R. Stencel.

Linear polarization and position angle were computed from Stokes $Q$ and $U$ using the formulation described in Bagnulo et al. (2009). We were careful to reverse the sign of Stokes $U$ as noted in the ESPaDOnS FITS header. Interstellar polarization was removed by Libre-ESpRIT, the data reduction pipeline for ESPaDOnS (Donati et al. 1997). Polarization data were binned (bin size $0.008 \mathrm{~nm}$ ) and bias-corrected. Spectra were corrected for F0 star stellar motion using the orbital solution from Stefanik et al. (2010). Polarization data were rotated to align instrument north with the rotation axis of the system as described by Kloppenborg et al. (2010).

We calculated the normalized flux equivalent width (EW) by integrating across the line. We corrected for small variations in the line normalization by subtracting an equivalent width, calculated from the featureless continuum adjacent to the line, and measured using the same wavelength span as the EW. We calculated integrated polarized flux EW by integrating $P=\sqrt{Q^{2}+U^{2}}$ across the line using the same wavelength span as the flux EW. We corrected for positive bias in the polarized flux by subtracting an integrated polarized flux EW also calculated using adjacent featureless continuum.

\section{Results and Conclusions}

Polarization increased dramatically during eclipse in species such as Fe I, Fe II, Ti II, Sc II and others (Geise et al. 2012). Polarization peaked around $3^{\text {rd }}$ contact for many species consistent with the observed increase in absorption. Persistent linear polarization was observed in several lines, including $\mathrm{H} \alpha$, even outside of eclipse.

$\mathrm{Fe}$ II $(4657 \AA$ and $4924 \AA$ ). The variability of the F0 star was evident in the photo-

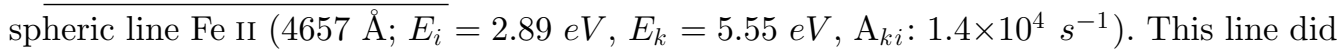
not exhibit increased absorption during eclipse (Strassmeier et al. 2014), but did exhibit out-of-eclipse linear polarization (Figs. 2 and 3). The Fe II $\left(4924 \AA ; E_{i}=2.89 \mathrm{eV}\right.$, $E_{k}=5.40 \mathrm{eV}, \mathrm{A}_{k i}: 4.3 \times 10^{6} \mathrm{~s}^{-1}$ ) line exhibited additional disk absorption, first redshifted in early eclipse and then blue-shifted in late eclipse (Fig. 4). The EW appeared to peak after mid-eclipse and an increase in polarized flux was also evident (Fig. 5). Although these lines have similar transition energies and arise from the same lower level, the longer wavelength line $(4924 \AA)$ is the stronger line. The Fe II (4731 $\AA E_{i}=2.89 \mathrm{eV}$, 

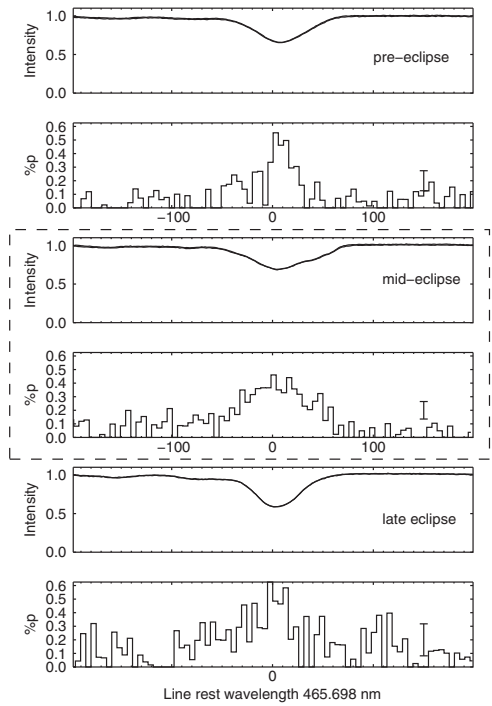
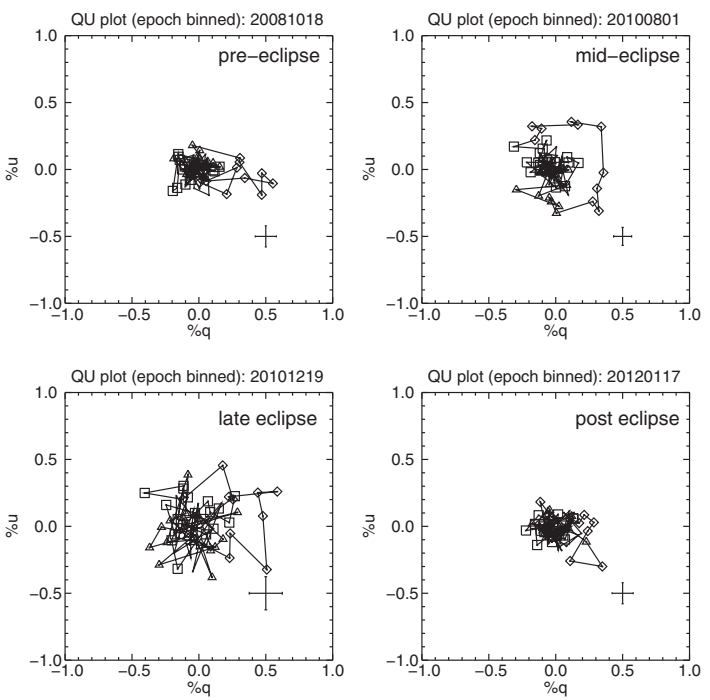

Figure 2. Line profiles and polarization (\%p) of the Fe II (4657 $\AA$ ) line at pre-eclipse, mid-eclipse and late eclipse epochs (left). Data are binned (bin size $0.008 \mathrm{~nm}$ ), centered on the line rest wavelength and corrected for stellar motion using the ephemeris from Stefanik et al. (2010). $Q U$-plots for binned data, rotated into the stellar frame (right). The blue wing (squares), red wing (triangles), and line core $\left( \pm 25 \mathrm{~km} \mathrm{~s}^{-1}\right.$, diamonds) polarization are shown. Loops in the $Q U$-plot arise from scattering by velocity-shifted material observed primarily in Stokes $U$ in the line wings. Average uncertainty in $\% \mathrm{q}$ and $\% \mathrm{u}$ indicated by crosses.
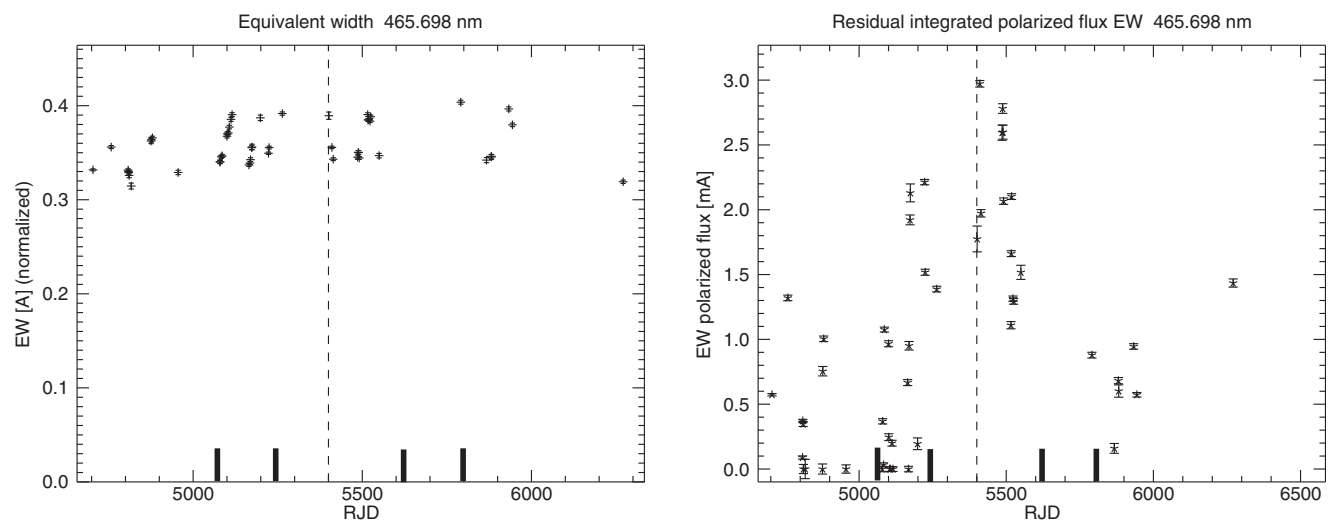

Figure 3. Equivalent width calculated from ESPaDOnS spectra by integrating across the Fe II (4657 $\AA$ ) line between $4656.0 \AA$ and $4658.3 \AA$, normalized to adjacent off-line continuum (left). The line is evidently variable, but no large increase in EW was observed during eclipse. Polarized flux integrated across the line, normalized by offline polarized flux EW (right). Scattered light may contribute to the increase in polarized flux and percent polarization $(\% p)$ observed during some eclipse epochs.

$\left.E_{k}=5.51 \mathrm{eV}, \mathrm{A}_{k i}: 2.8 \times 10^{4} \mathrm{~s}^{-1}\right)$ line, another in the multiplet, showed no additional disk absorption and exhibited similar polarization behavior as the weaker Fe II (4657 $\AA$ ) line.

K I (7699 A). Lambert \& Sawyer (1986) observed that the equivalent widths of K I

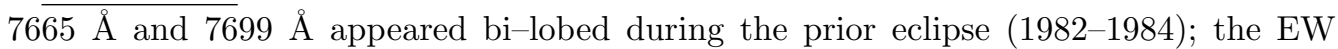
attained a maximum value just following the onset of totality ( $2^{\text {nd }}$ contact) and just 

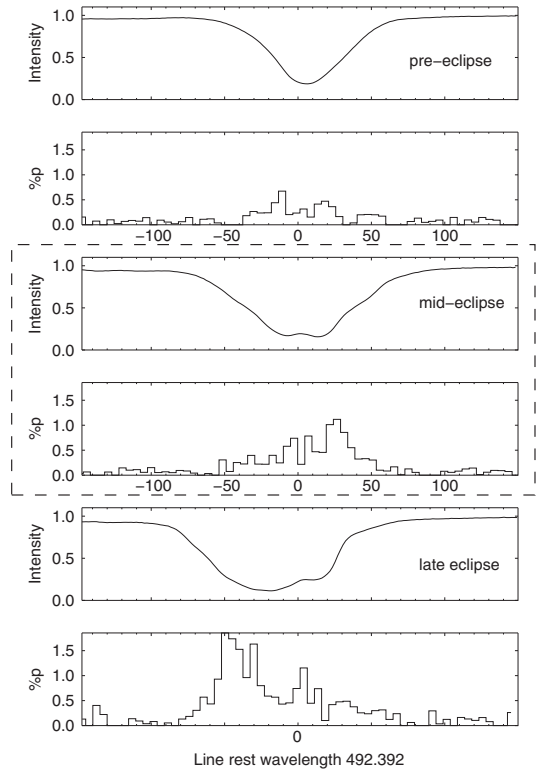
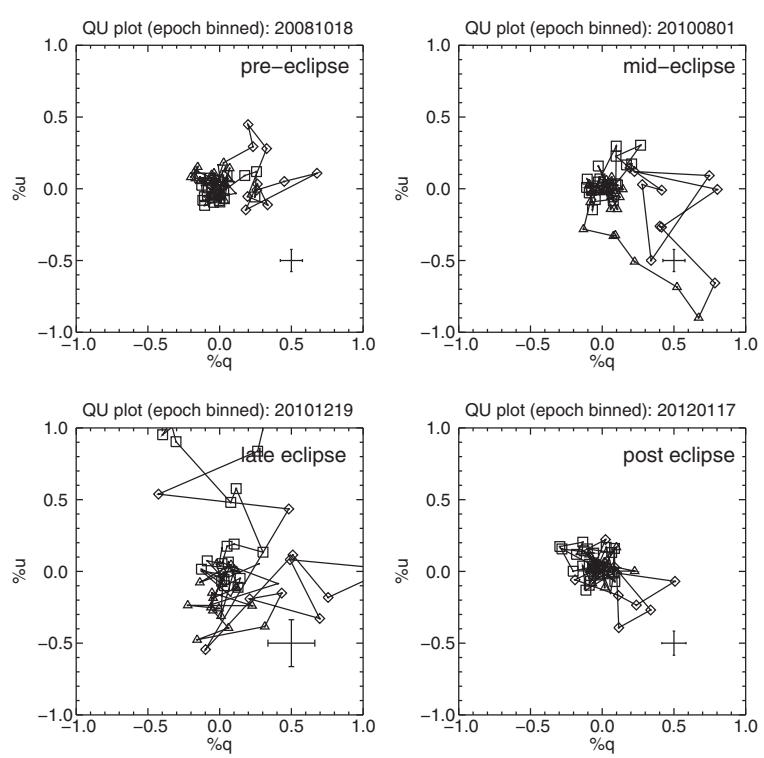

Figure 4. Line profiles and polarization (\%p) of the Fe II (4924 $\AA$ ) line at pre-eclipse, mid-eclipse and late eclipse epochs (left). Data are binned (bin size $0.008 \mathrm{~nm}$ ), centered on the line rest wavelength and corrected for stellar motion using the ephemeris from Stefanik et al. (2010). $Q U$-plots for binned data, rotated into the stellar frame (right). Symbols as in Fig. 2.
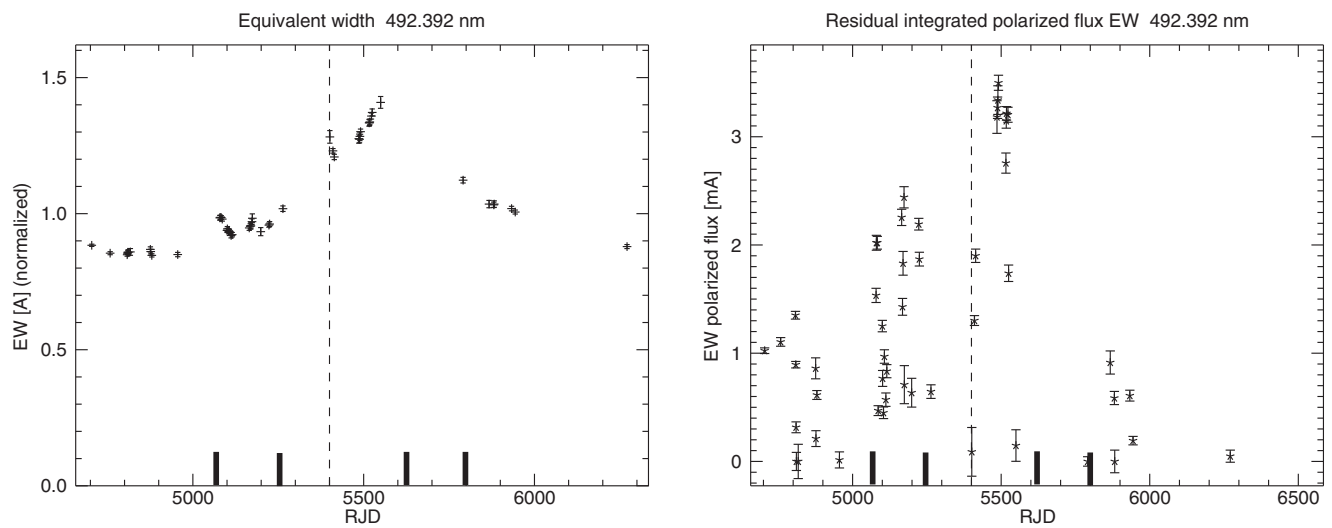

Figure 5. Equivalent width calculated from ESPaDOnS spectra by integrating across the Fe II (4924 $\AA$ ) line between $4922.0 \AA$ and $4926.5 \AA$, normalized to adjacent off-line continuum (left). The line is evidently variable, but no large increase in EW was observed during eclipse. Polarized flux integrated across the line, normalized by offline polarized flux EW (right). Compare this line to Fe II $4657 \AA$ (Fig. 3).

prior to the end of totality ( $3^{\text {rd }}$ contact). This line behavior was repeated during the most recent eclipse (Leadbeater et al. 2012, Strassmeier et al. 2014). The absorption line consisted primarily of an interstellar feature outside of eclipse. Our calculated equivalent widths closely resembled those by these observers (Fig. 6). Significant linear polarization (>3 sigma) was not observed in K I (7699 $\AA$ ) in our spectra until Nov 2010, more than 100 days after mid-eclipse, even though red-shifted absorption attributed to the disk was observed in the line near $1^{\text {st }}$ contact, in Sept 2009. The linear polarization returned to near zero by August 2011, our first observation after $3^{\text {rd }}$ contact. 

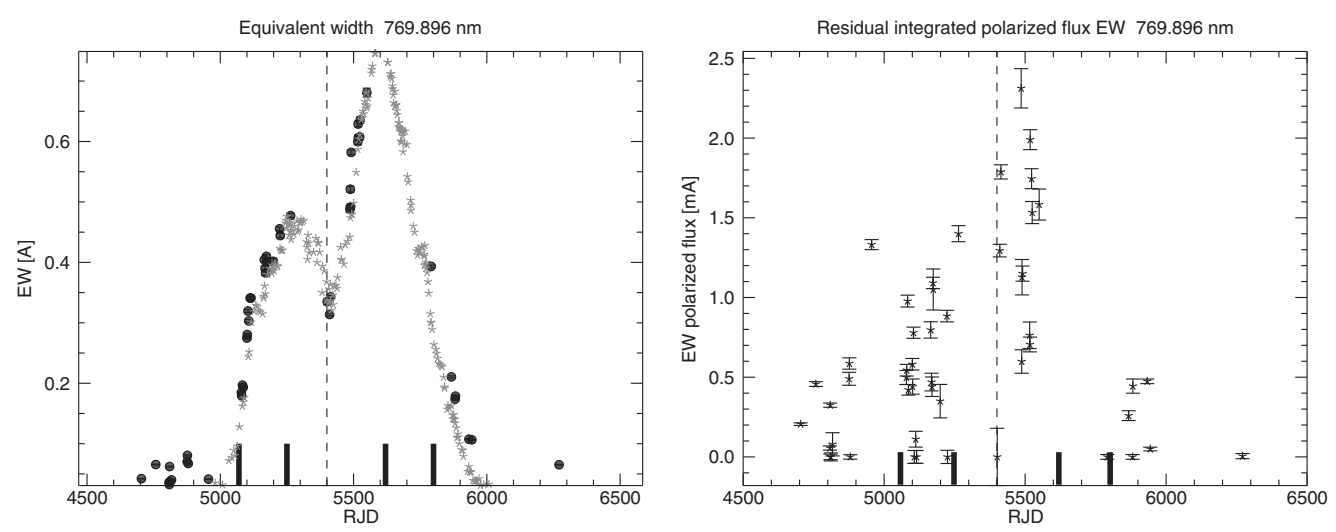

Figure 6. Integration of normalized flux across the K I (7699 Å) line by RJD (black circle) compared to results of Strassmeier et al. (2014) (grey). An interstellar component has been removed from the equivalent width. Mid-eclipse is marked by a vertical dashed line; $1^{\text {st }}, 2^{\text {nd }}$, $3^{\text {rd }}$ and $4^{\text {th }}$ contact are denoted by solid lines (left). Residual polarized flux integrated across the line, normalized by offline polarized flux (right). Note the increase in scattered light after mid-eclipse, near $3^{\text {rd }}$ contact. Also note the variable, presumed F0 star, absorption observed in the line out-of-eclipse.

$H \alpha(6563 \AA)$. Mauclaire et al. (2012) found the equivalent width of the $\mathrm{H} \alpha$ line to be anti-correlated with the observed $V$ band magnitude during eclipse. The EW increased as the $V$ magnitude decreased with the onset of eclipse and decreased as the system brightened after $3^{\text {rd }}$ contact. Our eclipse coverage had a 9-month gap beginning about 2 months before $3^{\text {rd }}$ contact and ending just prior to $4^{\text {th }}$ contact, but our EW data reflected similar variations with time as those of other observers. Compare the $\mathrm{H} \alpha \mathrm{EW}$ to the bi-lobed EW observed in K I (7699 $\AA)$. The linear polarization generally increased with increasing absorption, especially in the line wings, but we observed epochs of low integrated polarized flux during eclipse (Fig. 7).

Polarization associated with absorption lines (both line absorption, \%p, and polarized flux, P) revealed that gas is not distributed evenly in the disk. For example, the leading edge of the disk was observed to be less polarized than the trailing edge of the disk for many gas species, such as K I (7699 ̊), presented here. Some lines exhibited greater polarization for a brief time $(\sim 2$ months $)$ in spectra taken before $3^{\text {rd }}$ contact, similar to the continuum polarization (Cole 2012), possibly due to a localized increase in the number of scatterers. The behavior of $\mathrm{H} \alpha \mathrm{EW}$ and polarized flux differed from $\mathrm{K} \mathrm{I}$ (7699 $\AA$ ). The $\mathrm{H} \alpha$ EW did not appear bi-lobed and no large increase in polarized flux was observed in $\mathrm{H} \alpha$ near $3^{\text {rd }}$ contact. This suggests that the hydrogen gas may have a more extended distribution in the system. The variability observed during eclipse in polarized flux in atomic transitions such as K I may indicate the presence of sub-structure in the distribution of gas associated with the disk. We also found polarization signatures attributable to disk rotation (Geise et al. 2015, in prep.).

\section{Acknowledgements}

The authors are grateful for support of this work in part from a bequest in support of astronomy from the estate of William Herschel Womble. Based on observations obtained at the Canada-France-Hawaii Telescope (CFHT), which is operated by the National Research Council of Canada, the Institut National des Sciences de l'Univers of the Centre National de la Recherche Scientique of France, and the University of Hawaii. This research 

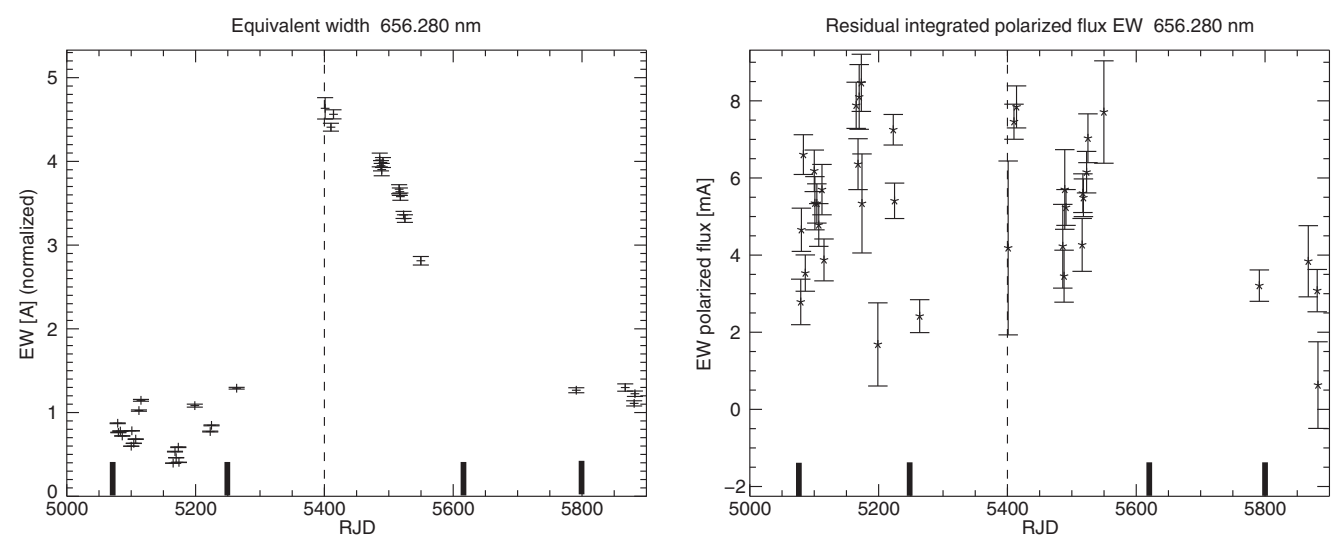

Figure 7. Equivalent width calculated from ESPaDOnS spectra by integrating across the $\mathrm{H} \alpha$ line between $6550 \AA$ and $6577 \AA$, normalized to adjacent off-line continuum; EW includes absorption and emission (left). The large velocity-shifted absorption contributed by the disk is demonstrated by the increase in EW by mid-eclipse. Polarized flux integrated across the line, normalized by offline polarized flux EW (right). Percent polarization $(\% p)$ increased during eclipse as the line deepened with additional disk absorption. Polarized flux appeared to increase after $1^{\text {st }}$ contact, but some eclipse epochs exhibited low polarized flux (e.g. near RJD 5200). No large increase in polarized flux was observed near $3^{\text {rd }}$ contact. Compare these observations to K I $(7699 \AA)$.

made use of the NIST Atomic Spectra Database. We would like to thank Nadine Manset, Jennifer Hoffman and Richard Pearson for helpful discussions.

\section{References}

Bagnulo, S., Landolfi, M., Landstreet, J. D., Landi Degl'Innocenti, E., Fossati, L., \& Sterzik, M. 2009, PASP 121, 993

Cole, G. M. 2012, The Journal of the American Association of Variable Star Observers 40, 787

Donati, J.-F., Semel, M., Carter, B. D., Rees, D. E \& Collier Cameron, A. 1997, MNRAS 291, 658

Donati, J.-F. 2003, in: J. Trujillo Bueno \& J. Sánchez Almeida (eds.), Proc. Solar Polarization (ASP-CS), 307, p. 41

Geise, K., Stencel, R. E., Manset, N., Harrington, D., \& Kuhn, J. 2012, The Journal of the American Association of Variable Star Observers 40, 767

Harrington, D. M. \& Kuhn, J. R. 2009, ApJS 180, 138

Hoard, D. W., Howell, S. B., \& Stencel, R. E. 2010, ApJ 714, 549

Kloppenborg, B., Stencel, R., Monnier, J. D., Schaefer, G., Zhao, M., Baron, F., McAlister, H., ten Brummelaar, T., Che, X., Farrington, C., Pedretti, E., Sallave-Goldfinger, P. J., Sturmann, J., Sturmann, L., Thureau, N., Turner, N., \& Carroll, S. M. 2010, Nature 464, 870

Lambert, D. L. \& Sawyer, S. R. 1986, PASP 98, 389

Leadbeater, R., Buil, C., Garrel, T., Gorodenski, S. A., Hansen, T., Schanne, L., Stencel, R. E, \& Stober, B. 2012, The Journal of the American Association of Variable Star Observers 40,729

Mauclaire, B., Buil, C., Garrel, T., Leadbeater, R., \& Lopez, A. 2012, The Journal of the American Association of Variable Star Observers 40, 718

Stefanik, R. P., Torres, G., Lovegrove, J., Pera, V. E., Latham, D. W., Zajac, J., \& Mazeh, T. 2010, AJ 139, 1254

Strassmeier, K. G., Weber, M., Granzer, T., Schanne, L., Bartus, J., \& Ilyin, I. 2014, AN 335, 904 